\title{
BMJ Open Quality Improving rates of implantable cardioverter defibrillator deactivation in end-of-life care
}

MR Javaid, Suzanne Squirrell, Fahad Farooqi

To cite: Javaid MR, Squirrell S, Farooqi F. Improving rates of implantable cardioverter defibrillator deactivation in endof-life care.BMJ Open Quality 2018;7:e000254. doi:10.1136/ bmjoq-2017-000254

Received 15 November 2017 Revised 7 March 2018 Accepted 5 April 2018
Check for updates

Barking, Havering \& Redbridge (BHR) NHS Trust, Queen's Hospital, Romford, UK

Correspondence to

Dr MR Javaid;

mahvesh.r.javaid@gmail.com

\section{ABSTRACT}

Implantable cardioverter defibrillators (ICDs) save lives in selected patients at risk of sudden cardiac death. However, in patents suffering with terminal illness, ICD therapy could pose a risk of unnecessary futile shocks which could lead to undignified discomfort in their final days of life. National guidelines advise that patients approaching the end of their natural life should be offered a compassionate choice of having their defibrillator deactivated. Following an actual clinical incident involving a patient receiving avoidable ICD shocks in his final hours, we identified shortcomings in communication and gaps in knowledge about ICD management in end-of-life care. We developed a quality improvement programme targeting training and educational support to general physicians and nurses at our large District General Hospital. A series of interventions were delivered including Grand Round presentation, departmental seminars and publicity posters. In parallel, we introduced a local protocol for implementing ICD deactivation which was published on our intranet for Trustwide accessibility. Following interventions, we examined the clinical notes of each end-of-life care patient who died with an ICD in situ over a 6-month observation period and recorded the proportion who received consent-guided ICD deactivation versus died with an active ICD in situ because no deactivation discussion had been offered. Before our interventions in 2015,0 out of 10 eligible patients $(0 \%)$ received consent-guided ICD deactivation. Six months into our campaign to encourage healthcare workers to undertake advance care planning discussion in 2016, 7 out of 13 eligible patients $(54 \%)$ received consent-guided ICD deactivation and no patients received shocks in their final month of life. This programme was successful in raising awareness of this emerging issue, improving physician knowledge and delivering patient choice as well as contributing to safe and compassionate end-of-life care.

\section{PROBLEM}

Healthcare workers are often unaware that dying patients who have previously been implanted with an implantable cardioverter defibrillator (ICD) are at significant risk of receiving futile shocks from their device, unless this function is specifically deactivated. This risk is neither rare nor trivial in ICD patients. There are very fewstudies which have described patients receiving shocks as they approach end of life. ${ }^{1-3}$ One study showed $19 \%$ of ICD patients received a shock in their last month and a further $8 \%$ in the last minutes of life. ${ }^{1}$

The number of patients approaching the end of their life with an active ICD or cardiac resynchronisation therapy defibrillator (CRT-D) is on the rise. ${ }^{4}$ More than $80 \%$ of ICD patients die in hospitals or healthcare facilities and two-thirds are treated in non-cardiac wards under the care of general or elderly care physicians who may lack knowledge of ICD clinical management. In a published multicentre cross-sectional study in Sweden, more than two-thirds of the physicians had experience of admitting ICD patients but rated their knowledge of ICD management to be low. ${ }^{5}$

In 2014, we received an informal complaint from a bereaved relative about an incident involving her terminally ill husband. The complainant had witnessed her husband receive futile repeated automated ICD shocks in his final hours of his life. He had a valid do not attempt resuscitation (DNAR) status, with the form completed by his responsible medical team, but they had not considered the impact of the implanted defibrillator which remained active and would thereby continue to detect and treat sustained ventricular arrhythmias with shocks. The attending healthcare workers observed the shocks and had tried in vain to intervene, but were unaware how to safely disable his device or who to contact for help after hours. This outcome might have been averted if the patient (and relatives) had been advised of this risk and prior consent obtained to undertake a planned deactivation of the shock function. An opportunity for such a discussion could have been undertaken during DNAR decision-making. The issue was compounded by a lack of awareness by his medical team of how to disable the device emergently, simply by applying a magnet over the pocket. This is safe to do even when the device is actively discharging. 
High-voltage electrical discharges from ICDs are known to be painful. In a conscious patient, it is usually described as feeling like a kick in the chest. It is particularly disturbing for relatives to witness as they are unlikely to have seen this before and would not be expecting this.

Our objective was to improve end-of-life care and patient experience with early ICD deactivation discussion with dying patients and planned 'in hours' deactivation of the device premortem in accordance with the patients' wishes (or best interests decision, if patient lacked capacity). Our target metric was to sustainably increase premortem consent-guided ICD deactivations in patients having end-of-life care planning and reduce the proportion of ICD deactivations after in-hospital death at our institution by at least $50 \%$ in 6 months.

\section{BACKGROUND}

ICDs and biventricular defibrillators save lives in selected patients at risk of sudden cardiac arrest due to ventricular arrhythmia. Their use is strongly evidence-based and supported by the National Institute for Health and Care Excellence guidelines as a cost-effective means to protect and prolong lives. ${ }^{6}$

In 2014/2015, around 9600 ICDs and CRT-Ds were implanted in the UK, ${ }^{7}$ yet UK implant numbers lag well behind Western European nations. In the UK, the number of implants is forecast to rise in line with broadening indications and improved access.

The most common indication for an ICD is for primary prevention in patients with severe heart failure who are at risk of sudden death due to ventricular arrhythmia. Heart failure is chronic and can progressively deteriorate, resulting in poor long-term prognosis. ICDs improve prognosis by treating life-threatening ventricular arrhythmia, but do not contribute positively to quality of life nor modify the disease progression. Most physicians and patients recognise that the shock function is usually inappropriate once the care plan shifts towards palliation, which may occur several years after the original implant.

Doctors need to have basic ICD knowledge or access to appropriate expertise if they are to provide the best possible care from implantation to end of life. The growing number of ICD patients will make it impossible for device specialists to follow all patients at all times which inevitably shifts some of the responsibility of ICD patient care to other specialties and primary care physicians.

Deactivating an ICD means disabling the shock function so the device no longer treats life-threatening arrhythmias such as ventricular fibrillation or ventricular tachycardia with a shock. The bradycardia pacing function of the ICD is unaffected, including resynchronisation therapy function (if available on the device). If ICD therapy is not deactivated, there is a significant risk of unnecessary futile shocks in the final month of life. This situation can be avoided by early identification of patients at risk (ie, expected to die within the next 12 months or have a DNAR decision in place).
The practicalities of hospital-based ICD deactivation mean that advance planning is necessary. Deteriorating patients (and their next of kin) should therefore be offered a discussion about ICD deactivation with the option to avoid the unnecessary and often inappropriate risk of painful shocks in the final moments of their life.

This discussion should take place as early as possible to allow sufficient time to effect the patients' wishes and be documented in clinical notes and be circulated to the general practitioner (GP), palliative care, heart failure nurse and pacing notes. It is also important to explain to patients that ICD deactivation is easy to perform, painless and will not cause death or accelerate demise from their underlying disease.

Our local Trust pathway for ICD deactivation mandates that patients' consent or best interests decision from healthcare professional (if a patient is deemed to lack mental capacity) is necessary prior to the process of physiologist-led deactivation. Out-of-hours emergency 'inactivation' of ICD shock function is achievable by application of a magnet over the device pocket, but is only effective as long as the magnet is not removed so this should be converted to a deactivation by a cardiac physiologist as soon as available.

British Heart Foundation guidelines (2013) state, 'The appropriateness of maintaining device therapy must be regularly reviewed as part of monitoring of the patient's progressive disease trajectory if there is any change in clinical status including the development of a life-limiting disease'. ${ }^{4}$ Joint guidance from Resuscitation Council UK, Heart Rhythm UK/Arrhythmia Alliance and British Cardiovascular Society advises that patients approaching end of life should be offered a choice of defibrillator deactivation to avoid shocks in the latter stages of their illness. ${ }^{8}$

Deactivating ICDs is legally and ethically justified as part of a compassionate end-of-life care plan. In legal terms, ICD deactivation is considered a withdrawal of medical treatment, it should not be confused with the act of 'assisting death' which is unlawful in the UK.

Barking, Havering and Redbridge University Hospitals NHS Trust cares for a population of around 800000 . We estimate that 50-100 new patients in our catchment population will have an ICD implanted each year, we anticipate a higher average prevalence of previously implanted patients for primary prevention in heart failure as our Trust serves an older demographic than the national average.

\section{BASELINE MEASUREMENT}

Between January 2015 and August 2015, we carried out an analysis of Queens Hospital mortality records and discovered 13 patients with an implanted defibrillator (standalone ICD or biventricular-ICD device) had died in our hospital without premortem deactivation. Also, 10 out of the $13(75 \%)$ deaths were expected in verified 'DNAR' patients yet the clinical notes indicated that in none of 
PRIMARY DRIVERS

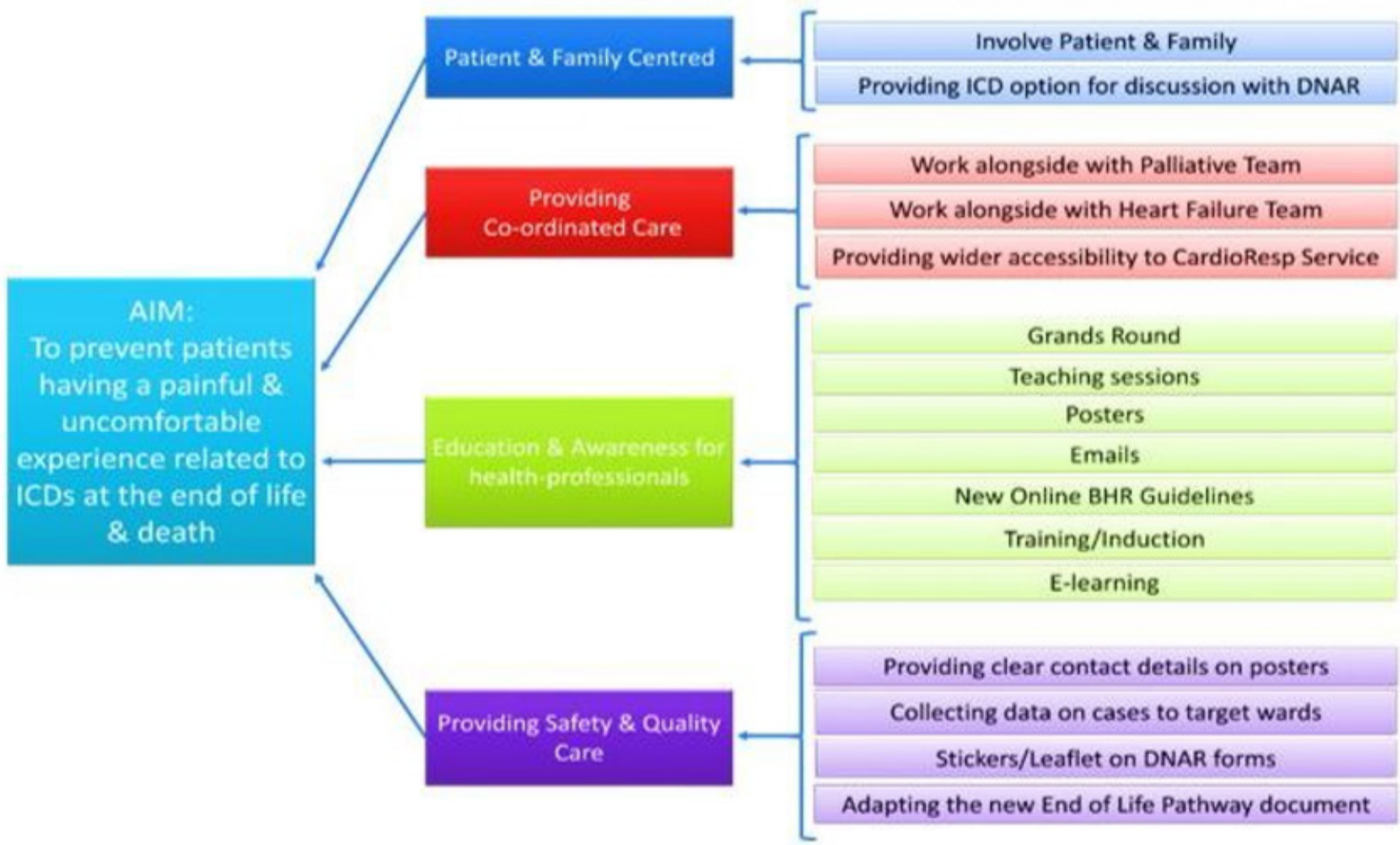

Figure 1 Driver diagram showing the primary and secondary outcomes following specific interventions. BHR, Barking, Havering \& Redbridge NHS Trust; DNAR, do not attempt resuscitation; ICD, implantable cardioverter defibrillator.

these cases had a discussion taken place with patients (or relatives) to offer deactivation exposing them to potential risk of futile shocks. This outcome represented a baseline $100 \%$ 'missed opportunity' rate by failing to offering discussions in eligible dying patients.

We undertook a preliminary 10-question staff survey in January 2016 to assess the level of awareness on the issue of ICD management in end-of-life care. This survey was undertaken by 100 health professionals which involved 80 general medicine physicians of all grades and 20 medical ward-based nurses and matrons representing most medical specialties (figure 1).

\section{DESIGN}

We established specific interventions supported by evidence from our staff survey (figure 1). The survey had identified gaps in knowledge about ICD device identification, confusion about function and lack of awareness about the local deactivation pathway, so we especially focused on these aspects in the teaching programme targeted at general physicians and medical ward nurses.

In our study, all patients who died between February 2016 and July 2016 with an active ICD at Queens Hospital, Romford, were included. Our investigation would compare the frequency of two mutually exclusive patient scenarios involving DNAR cases who had died in hospital. The first group was DNAR patients who had died with an active ICD (without any deactivation discussion or planning) versus the second group which comprised DNAR patients who had their ICD deactivated (by patient consent or best interests decision) or left active (due to patients' wishes) before dying. Every DNAR inpatient at Queens Hospital who had died $>24$ hours after admission with their ICD still left active because no advance planning discussion had taken place was deemed a missed opportunity for ICD deactivation as it potentially exposed a dying patient to unnecessary shocks.

From February 2016, we carried out targeted multifaceted interventions each month aimed at increasing awareness and knowledge on ICD management in end-of-life care such as Grand Round presentation, departmental seminar teaching, publicity posters (figure 2), including ICD deactivation discussion integrated into the end-oflife care pathway. We then studied the short-term impact of these exercises on ICD deactivation rates.

\section{STRATEGY}

Every month, after each Plan-Do-Act-Study (PDSA) cycle, the number of ICDs deactivated premortem versus postmortem were recorded from the Cardiorespiratory Unit's database at Queens Hospital, Romford, Essex. The comprehensive database records all deactivations carried out at Queens Hospital by cardiac physiologists. 

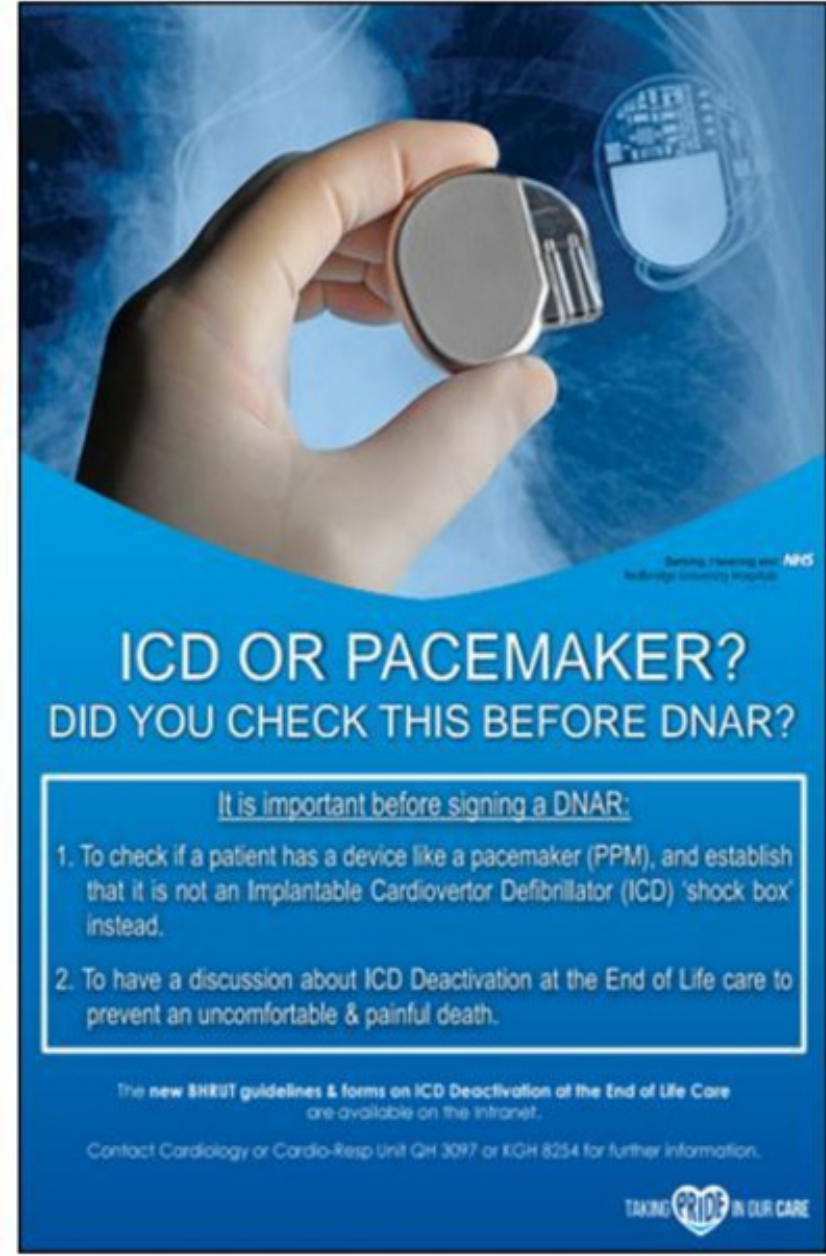

Figure 2 The poster displayed across the wards and on our Trust intranet. DNAR, do not attempt resuscitation.

We co-authored (in collaboration with palliative care colleagues) a special paragraph in the updated Trust guidance pathway on end-of-life care to remind clinicians about ICD management at the time of planning DNAR decisions. Our Trust guidance on ICD deactivation in patients approaching end of life was formally introduced in January 2016, so we decided to monitor starting in February 2016.

We looked at all deactivations between February 2016 and July 2016 including inpatients and outpatients and analysed data on how we were performing against our stated target (figure 1). Following each PDSA cycle, the data were collected retrospectively for analysis. Case notes were recalled for verification, audit and quality improvement purposes. During this quality improvement project, four PDSA cycles took place.

\section{PDSA cycle 1}

Raising awareness and improving knowledge among general physicians were the main goals for the first cycle. We presented the major issue and the guidelines at many different departmental meetings including intensive care unit (ICU), anaesthetics and accident and emergency (A\&E), and at the Trust's multidisciplinary meetings. We often used visual aids, for example, taping a magnet onto 'Little Anne' cardiopulmonary resuscitation (CPR) mannequin to demonstrate how to inactivate ICDs in an emergency. Each presentation was well attended by a wide range of medical specialties, with many questions answered, and positive feedback was obtained.

\section{PDSA cycle 2}

During this cycle, we reached out to as many staff as possible using electronic communications to staff members and groups which were unable to physically attend the first cycle of teaching events. This provided an opportunity to raise awareness to a wide audience through informative e-messages via Trust email and weekly Trust e-newsletter.

\section{PDSA cycle 3}

This cycle was intended to reinforce the message and help plan deactivation of ICDs in end-of-life care. We developed an informative poster which was displayed at strategic sites across many wards and departments to provide an 'aide memoire' for healthcare professionals.

\section{PDSA cycle 4}

The last cycle was targeted at offering teaching for staff who worked on medical wards which had a history of patients with missed opportunities to deactivate ICDs at end of life identified from our baseline analysis. We also created a checklist to help staff distinguish whether an implanted electronic cardiac device was an ICD or a pacemaker.

\section{RESULTS}

With the preliminary staff survey, the results revealed $>60 \%$ of medical staff surveyed were unaware of the consequences of not discussing and planning ICD management with patients (and their relatives) nearing the end of their life. Here are some example results for some specific question from the survey

- Do you know how to identify if your patient has an ICD? No $54 \%$.

- Have you ever seen or participated in a discussion about ICD deactivation involving a patient approaching the end of their life? No $65 \%$.

- Do you know when to discuss ICD deactivation with a patient or relative? No $68 \%$.

- Do you know how to deactivate an ICD in an emergency? No $72 \%$.

\section{Baseline analysis}

Over the 8-month period in 2015 (before undertaking PDSA cycles), none of the 10 eligible dying patients had received ICD deactivation discussions.

\section{PDSA cycle 1}

Two out of four patients had their ICD deactivated before death. 


\section{Percentage of Deaths with an active ICD in-situ}

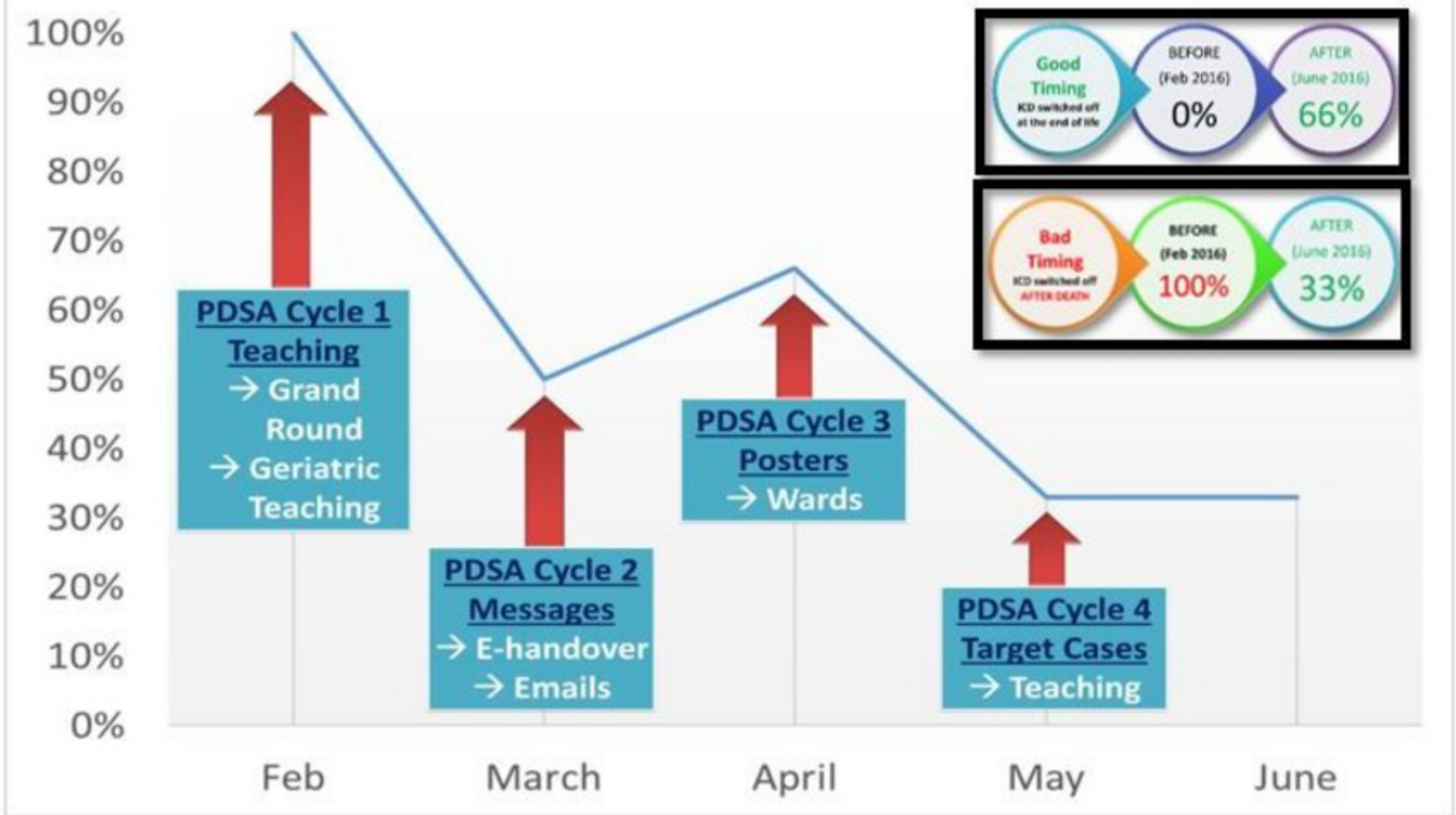

Figure 3 Run chart showing outcomes of interventions expressed as percentage of cases with active implantable cardioverter defibrillator (ICD) in situ postmortem. PDSA, Plan-Do-Study-Act.

\section{PDSA cycle 2}

One out of three patients died with their ICD deactivated, whereas the other two patients had died without prior discussion and necessitated postmortem deactivation.

\section{PDSA cycle 3}

Two out of three patients had died with advance planning to deactivate their ICD before death.

\section{PDSA cycle 4}

Two out of three patients had their ICD deactivated before death.

In all four PDSA cycles of our study, all DNAR patients offered a choice about their ICD management elected to have a deactivation while on an end-of-life care pathway. Over the 6-month monitoring period, 7 out of the total 13 patients had received a consent (or best interests, if lacking capacity)-guided ICD deactivation and no patients received a shock from their device in their final month of life (figure 3).

\section{LESSONS}

The decision not to attempt resuscitation is not the same as a decision to deactivate an ICD; the former is a medical decision usually made by the physician in discussion with the patient involved and/or next of kin, but the latter mandates the consent of the patient if they have capacity. ${ }^{8}$ It would be improper to assume all dying patients will give consent to have their ICD deactivated-it is important to check and act in accordance with the patient's wishes, to do otherwise could contravene national guidance ${ }^{8}$ and risk legal repercussions. All decisions require careful assessment of each individual situation. A patient may wish to be considered for CPR despite choosing to have their ICD deactivated ${ }^{9}$ or vice versa, the patient may not want CPR, but keep their ICD remain activated. ${ }^{8}$

Discussions about ICD deactivation have to be handled sensitively and are best handled by staff trained in higher communication skills. It is the role of the physician to help the patient think about the option of deactivation in the context of their goals of care. A patient can make whatever choice they wish, but while it is often consistent to choose DNAR but leave the device on, it is almost never consistent with a patient's goals of care to choose deactivation but still want CPR. The British Heart Rhythm Society (HRS) guidelines (2014) states, 'These choices must be respected and kept under review with the opportunity for decisions to be changed as the person's condition progresses'. ${ }^{9}$

Therefore, the deactivation of ICDs potentially raises ethical and legal dilemmas. ${ }^{10}$ According to the guidelines and recommendations of these arrhythmology societies, withholding or withdrawing of life-sustaining treatments 
from terminally ill patients who do not want the treatments is ethical and legal and 'should be honoured in accordance with the legally and ethically established rights of patients". ${ }^{5}$ The European Heart Rhythm Association and HRS consensus statements (both published in 2010) discuss the ethical and legal aspects in more depth. ${ }^{45}$

Having guidelines is useful, but to be clinically effective they need to be understood, retained and implemented by those who care for patients' A\&E, clinic and ward settings. After completing our quality improvement project, an update to national guidelines ${ }^{11}$ was published by the British Society of Cardiology and National Council for Palliative Care. Such guidelines can help hospitals to prepare for this real and growing risk.

The rationale for discussing deactivation is not just to follow guidelines or avoid legal risk, but because patient autonomy is a cornerstone of medical ethics, and offering palliation-focused care at end of life is the compassionate approach.

\section{LIMITATIONS}

The main limitation of this study is that it is a single-centre study with a small sample size, so statistical testing of the data is not appropriate. The observed outcomes may not be transferable to other institutions as data were captured for a relatively short duration and any change in the month following an intervention cannot be proven to be due to the preceding intervention. Every DNAR inpatient at Queens Hospital who had died $>24$ hours after admission with their ICD still left active because no advance planning discussion had taken place was deemed a missed opportunity for ICD deactivation as it potentially exposed a dying patient to unnecessary shocks, but there can be unknown factors that might have legitimately prevented a deactivation discussion, for example, inability to contact next of kin.

Our preliminary 10-question staff survey in January 2016 showed that level of awareness of ICD management in end-of-life care was poor. The survey had identified gaps in knowledge about ICD device identification, confusion about function and lack of awareness about the local deactivation pathway, so we especially focused on these aspects in the teaching programme targeted at general physicians and medical ward nurses.

In order to sustain awareness of ICD management issues in end-of-life care, we found synergistic interventions needed to be rolled out over a protracted period to have a sustained impact, otherwise there was a risk that the original message would not be retained or displaced by other priorities. When patients get an ICD implanted, cardiologists need to prewarn patients that they may choose deactivation in the future if there is a deterioration in physical health and quality of life. ${ }^{11}$ DNAR discussions provide a good opportunity to discuss ICD deactivation. If patient consents, then ICD deactivation should not be delayed as protection from unnecessary shock is immediate. It can even be reversed if the patient changes their mind. Our existing Trust DNAR form does not have a specific section to document ICD deactivation discussion outcome. We have recommended this addition and hope to have it implemented.

We did not include patients with an ICD who died in the community. It is similarly important if there is a community DNAR in place, that the GP, community heart failure team and 'hospice at home' team ensure that the patient has their ICD deactivation discussion in advance to allow sufficient time to arrange an elective deactivation, if the patient agrees. $^{12}$

\section{CONCLUSION}

This quality improvement programme involved a series of small interventions designed to raise staff knowledge and awareness of ICD management issues in dying patients and increase conversations about ICD deactivation.

The programme was tested for effectiveness through improvement cycles. This translated into significant outcome improvements, namely increased numbers of ICD discussions and premortem deactivations and consequently proportionate decrease in avoidable ICD postmortem deactivations which was sustained over 6 months. Before our interventions in 2015, 0 out of 10 eligible patients $(0 \%)$ received consent-guided ICD deactivation, by the end of our programme in 2016, 7 out of 13 eligible patients (54\%) received consent-guided ICD deactivation. All DNAR patients offered a choice about their ICD management elected to have a deactivation. These measures were coordinated and implemented safely and are an example of good quality, patient-centred care being offered at our Trust.

Our endeavour was successful in achieving the desired objective-no further patients received avoidable shocks during the 6-month observation period and many more inpatients received consent-guided ICD deactivation following a DNAR order. This was a relatively short duration, single-centre pilot study with relatively small numbers of patients, so no statistical testing was applied, but we have been able to demonstrate that fewer unwanted ICD shocks in patients approaching the end of their natural life are achievable through education and supervised training. These findings are very encouraging but this programme needs to be tested on a larger scale and is likely to require regular ongoing educational support in order to sustain the positive impact.

Funding This research received no specific grant from any funding agency in the public, commercial or not-for-profit sectors.

Competing interests None declared.

Provenance and peer review Not commissioned; externally peer reviewed.

Open Access This is an Open Access article distributed in accordance with the Creative Commons Attribution Non Commercial (CC BY-NC 4.0) license, which permits others to distribute, remix, adapt, build upon this work non-commercially, and license their derivative works on different terms, provided the original work is properly cited and the use is non-commercial. See: http://creativecommons.org/ licenses/by-nc/4.0/ 
(c) Published by the BMJ Publishing Group Limited. For permission to use (where not already granted under a licence) please go to http://www.bmj.com/company/ products-services/rights-and-licensing/

\section{REFERENCES}

1. Sherazi S, McNitt S, Aktas MK, et al. End-of-life care in patients with implantable cardioverter defibrillators: a MADIT-II substudy. Pacing Clin Electrophysiol 2013;36:1273-9.

2. Padeletti L, Arnar DO, Boncinelli L, et al. EHRA Expert Consensus Statement on the management of cardiovascular implantable electronic devices in patients nearing end of life or requesting withdrawal of therapy. Europace 2010;12:1480-9.

3. Lampert R, Hayes DL, Annas GJ, et al. HRS Expert Consensus Statement on the Management of Cardiovascular Implantable Electronic Devices (CIEDs) in patients nearing end of life or requesting withdrawal of therapy. Heart Rhythm 2010;7:1008-26.

4. BHF. ICD deactivation at the end of life: Principles and practice. 2013 https://www.bhf.org.uk/-/media/files/publications/hcps/icddeactivation.pdf (accessed 30 Aug 2016).

5. Kinch Westerdahl A, Frykman V. Physicians' knowledge of implantable defibrillator treatment: are we good enough? Europace 2017;19:1163-9.
6. National Institute for Health and Care Excellence. Implantable cardioverter defibrillators and cardiac resynchronisation therapy for arrhythmias and heart failure. 2014 https://www.nice.org.uk/ guidance/ta314 (accessed 1 Nov 2017).

7. Surrey Cardiac Physiologists' Network. Guidelines for deactivating implantable cardioverter defibrillators (ICDs) in people nearing the end of their life. 2013 http://www.slcsn.nhs.uk/files/cardiac/slguidelines-icd- deactivation-012013.pdf (accessed 1 Nov 2017).

8. Resus Council. Decisions relating to cardiopulmonary resuscitation. 2014 https://www.resus.org.uk/EasySiteWeb/GatewayLink.aspx? alld=838 (accessed 30 Aug 2016).

9. BHRS. Deactivation of implantable cardioverter defibrillators towards the end of life. 2015 http://www.bhrs.com/files/files/Guidelines/ CIEDs_Deactivation.pdf (accessed 30 Aug 2016).

10. Floria M, Rezuș C, Ciutea MP, et al. Implantable cardioverter defibrillator deactivation in patients nearing the end of life. Romanian Journal of Bioethics 2012;10:134-40.

11. Pitcher D, Soar J, Hogg K, et al. Cardiovascular implanted electronic devices in people towards the end of life, during cardiopulmonary resuscitation and after death: guidance from the Resuscitation Council (UK), British Cardiovascular Society and National Council for Palliative Care. Heart 2016;102(Suppl 7):A1-A17.

12. Fernando J, Percy J, Davidson L, et al. The challenge of providing palliative care to a rural population with cardiovascular disease. Curr Opin Support Palliat Care 2014;8:9-14. 